\title{
Polinización de Mucuna mutisiana (Kunth) D.C. por murciélagos Glossophaga soricina y Glossophaga longirostris (Phyllostomidae: Glossophaginae) en el Bosque Seco Tropical (BsT) al norte de Colombia
}

\author{
Pollination of Mucuna mutisiana (Kunth) D.C. by \\ bats Glossophaga soricina and Glossophaga longirostris \\ (Phyllostomidae: Glossophaginae) in the Tropical \\ Dry Forest (BsT) in Northern Colombia
}

\begin{abstract}
Meriele Rebolledo Contreras ${ }^{1}$
${ }^{'}$ Magister en Ciencias Naturales, investigadora del Departamento de Química y Biología de la Universidad del Norte, Barranquilla, Colombia.

Email:merieler@uninorte.edu.co

Orcid: oooo-0oo3-3205-2334
\end{abstract}

\section{Resumen}

Los sistemas de polinización biótica se pueden considerar como un proceso ecológico donde la interacción entre plantas y polinizadores determina la reproducción de las primeras, además, asegura la variabilidad genética debido a la transferencia del polen entre diferentes plantas, dado por la polinización cruzada (Aguilar et al. 2006; Ashman et al. 2004). Se estima que los murciélagos son agentes polinizadores de un amplio grupo de plantas en el trópico, favoreciendo la transferencia del polen a largas distancias entre plantas con flores. El objetivo de esta investigación fue describir la biología de polinización de M. mutisiana (Kunth) DC., una fabácea polinizada por murciélagos glosofaginos en fragmentos de Bosque seco Tropical (BsT) de la región Caribe de Colombia. Los resultados muestran que estas plantas florecen entre los meses de marzo y mayo y de octubre hasta noviembre, con una duración aproximada de ocho a 14

\section{Historia del artículo}

Fecha de recepción:

19-06-2021

Fecha de aceptación:

19-10-2021

DOI: https://doi.

org/10.47374/

novcol.2021.v16.1945 
semanas. Su antesis es crepuscular, con la mayor producción de flores entre la segunda y tercera semana de floración, con un promedio de 57 a 108 racimos, con cinco a seis flores maduras abiertas por noche por racimo y con un promedio de 30 flores por individuo. Para que ocurra la polinización de M. mutisiana en el BsT, se requiere un movimiento brusco que activa el mecanismo de apertura de los pétalos de la flor y posterior liberación del androceo y del gineceo, necesaria para su reproducción. Las flores tienen un tamaño de $6.3 \mathrm{~cm}(\mathrm{SD}=0.18 ; \mathrm{N}=25)$, con picos máximos de producción de néctar por noche de $18.58 \mu \mathrm{l}(\mathrm{SD}=18.74 ; \mathrm{n}=112) \mathrm{y}$ los máximos porcentajes de concentración de azúcar fueron de $4.68^{\circ} \mathrm{Brix}(\mathrm{SD}=5.03$; $\mathrm{n}=112$ ) a las 18:00 h. Dos potenciales polinizadores, los murciélagos Glossophaga soricina (Pallas 1766) y Glossophaga longirostris (Miller, 1898), visitan las flores de M. mutisiana, con un pico máximo de visita de $34.25 \%(\mathrm{SD}=39.8 ; \mathrm{n}=112)$ desde las 19:0o h a las 21:00 h. También se observó que los colibríes (Antracotoras nigricolis y Phaetornis sp.) visitan las flores durante el día, por la noche se observaron otros visitantes como la zarigüeya (Caluromys $s p$.) y las polillas nocturnas de la familia Sphingidae. Todas estas observaciones confirmaron el síndrome de polinización de quiropterofilia. Sus rasgos florales de atracción, e incluso de recompensas florales de $M$. mutisiana, presentan congruencias entre la utilización del recurso floral y capacidad de forrajeo de sus principales polinizadores que se evidencia al efectuarse el contacto entre las anteras y los pistilos con la parte anteroposterior del cuerpo de los individuos de este grupo polinizador en el BsT, Así mismo, se encontró un alto valor en las relaciones que estos murciélagos tienen con los recursos ofrecidos por el entorno, dada su flexibilidad en la dieta, favoreciendo no solo los sistemas de polinización cuando hay disponibilidad de néctar, sino también por que combinan su dieta nectarívora con frutos e insectos, permitiendo la coexistencia de estos dos potenciales polinizadores de M. mutisiana.

Palabras clave: Sistemas de polinización biótica, quiropterofilia, Bosque Seco Tropical, biología de polinización.

\section{Abstract}

Biotic pollination systems can be considered as an ecological process where the interaction between plants and their pollinators determines plant reproduction. It also ensures genetic variability due to pollen transfer between different plants because of cross pollination. It is estimated that bats are pollinating agents to a wide group of plants in the tropics, favoring the transfer of pollen over long distances in plants with flowers that have adaptations for pollination by chiropterans. The objective of my research consisted in describing the biology of pollination of M. mutisiana (Kunth) DC., a fabaceous plant pollinated by swee-tooth bats, in Tropical Dry Forests in the Caribbean region of Colombia. In addition to the possible incidence of the ecological role of nectarivores bats in the dynamics of the Tropical Dry Forest. The results on 
its phenology shows that the anthesis is twilight starting at 18:00 $\mathrm{h}$. between the months of March and May; and then again October through November, with an approximate duration of 8 to 14 weeks. The maximum flower production happens between the second and third week of flowering with an average of 57 to 108 clusters; 5 to 6 mature flowers opening per night/cluster; and an average of 30 flowers per individual. It was observed that for $M$. mutisiana pollinisation in BsT to occur, a sudden movement is required to activate the flower opening mechanism towards the subsequent release of the necessary androecium and gynoecium for their reproduction. The flowers size is $6.3 \mathrm{~cm}$ $(\mathrm{SD}=0.18 ; \mathrm{N}=25)$, with maximum peaks of nectar production per night of around $18.58 \mu \mathrm{l}(\mathrm{SD}=18.74 ; \mathrm{n}=112)$. Maximum percentage of sugar concentration was $4.68^{\circ}$ Brix $(\mathrm{SD}=5.03 ; \mathrm{n}=112)$ at 6:00 p.m. Two potential pollinators bats: Glossophaga soricina (Pallas 1766) and Glossophaga longirostris (Miller, 1898), visit the flowers of M. mutisiana, with a maximum visit peak of $34.25 \%(\mathrm{SD}=39.8 ; \mathrm{n}=112)$ from 19 : oo a.m. to 9:0o p.m. It was also observed that daylight visits were mostly made by hummingbirds (Antracotoras nigricolis and Phaetornis sp.), At night, other visitors such as opossums (Caluromys sp.) and nocturnal moths of the Sphingidae family were observed. All these observations confirmed the chiropterophilic pollination syndrome in these plant species. Its floral features of attraction and even floral rewards of M. mutisiana showed congruences between the use of the floral resource and the foraging capacity of its main pollinators, which is evidenced by contact between the anthers and pistils with the anteroposterior part of the body of the individuals of this pollinating group In the BsT. Likewise, a high value was found in the relationships that these bats have with the resources offered by the environment, given their flexibility in the diet favoring, not only the pollination systems when there is availability of nectar, but also by combining its nectarivores diet with fruits and insects, allowing these two potential pollinators of M. mutisiana to coexist.

Keywords: Biotic pollination systems, chiropterophily, Tropical Dry Forest, pollination biology.

\section{Introducción}

Se estima que los sistemas productivos agropecuarios y la deforestación, producen aislamiento espacial del hábitat, lo cual tiene efectos negativos, no solo sobre el desgaste del suelo, sino también sobre los servicios ecosistémicos que los bosques proveen (Aguilar et al. 2006, 2019; Carbone et al. 2019). Sobrellevar estos efectos negativos se ha convertido en un desafío importante para la conservación del Bosque seco Tropical (BsT) y una de las principales amenazas para la biodiversidad (Pizano et al. 2014). Entender los procesos ecológicos como son los sistemas de polinización permite esclarecer cómo responden las plantas a los efectos negativos de carácter antrópico, tanto en ecosistemas naturales como en los agroecosistemas productivos (Aguilar et al. 2006, 2019; Aldana-Domínguez et al. 2017; Cusser, Neff, y Jha 2016). Más aun 
cuando los estudios sobre los servicios de polinización permiten entender uno de los mecanismos más importantes para asegurar las variabilidad genética en las angiospermas como es el caso de la polinización cruzada, que implica la transferencia del polen entre diferentes flores (Ashman et al. 2004; Chacoff, Aizen, y Aschero 2008).

Sumado a esto, los estudios enfocados en las adaptaciones que presentan las plantas polinizadas por murciélagos han cobrado importancia, porque en esta interacción actúan como vectores de polen óptimo que obtienen de una fuente de néctar óptima (Horner, Fleming, y Sahey 1998; Voigt 2004) Leptonycteris curasoae (= L. sanborni. Además, las plantas de este género en su trayectoria evolutiva han adquirido un conjunto de características propias en las estructuras de las flores para ciertos vectores de polen, lo cual permite inferir la forma como se produce el traslado de polen entre las flores, basado en el supuesto de la teoría del síndrome de polinización.

En este sentido, el propósito de mi investigación fue describir la polinización de M. mutisiana (Kunth) DC., una fabácea polinizada por murciélagos, a partir de información recopilada sobre la biología floral, disponibilidad del néctar en las flores y la frecuencia de visita de los murciélagos de la subfamilia Glossophaginae, como una aproximación a la teoría de los síndromes de polinización, dado que esta especie presenta un "conjunto de rasgos florales de atracción e incluso de recompensas florales, asociados con la utilización y capacidad de forrajeo de un grupo específico de polinizadores"(Faegri y Pijl 1979; Fenster et al. 2004; Willmer 2011). Entender cómo incide el rol ecológico de los murciélagos nectarívoros en la dinámica del bosque seco tropical, dada la biología de polinización de M. mutisiana, permite identificar un modelo para explorar la utilización del recurso alimenticio por parte de sus polinizadores y que sustente la adaptación en un sistema de polinización para mamíferos voladores dependientes y/o generalistas. Los rasgos atractivos de las flores de M. mutisiana permiten documentar estudios enfocados en la dirección y regulación de la interacción de estas con sus potenciales polinizadores.

Mucuna mutisiana (Kunth) DC., es una de las especies tropicales del género que habita en bosques primarios, de galería, secundarios e intervenidos en los bordes de los ríos desde el nivel del mar hasta los 2500 msnm, en Centro y Suramérica (T. M. D. Moura et al. 2018). Es conocida en Colombia como "Cogolo", "Ojo de buey", "Ojo de venado" o "Pica pica". Sus rasgos comprenden flores de color verdeamarillentas o amarillo claro, agrupadas en racimos de flores sobre pedúnculos que bajan del dosel a lugares abiertos de hasta $80 \mathrm{~cm}$ de longitud. Estas características son adecuadas para la atracción, acceso y visita de sus polinizadores y poseen una estrecha relación con la teoría del síndrome de polinización chiropterofilico (Faegri y Pijl 1979; Kobayashi et al. 2015, 2018; T. M. Moura et al. 2016).

Esta investigación se centra en la biología de la polinización y la relación entre el patrón de producción de néctar y la 
concentración de azúcar con respecto a la frecuencia y comportamiento de los visitantes florales, lo que a su vez ayudará a definir la diversidad de polinizadores mamíferos en el BsT y su importancia adaptativa a estas características florales en dichas interacciones tróficas. No obstante, con la finalidad de esclarecer el sistema de polinización de M. mutisiana he planteado los siguientes objetivos: 1) Caracterizar la biología floral. 2) Describir la producción de néctar y concentración de azúcar en el néctar. 3) Identificar los visitantes florales y descripción del comportamiento de las flores de Mucuna mutisiana (Kunth) DG. 4) Establecer la relación de los patrones de visita de los visitantes de las flores de $M$. mutisiana con la producción de néctar y la concentración de azúcar de esta planta.

\section{Materiales y Métodos}

\section{Área de estudio}

Este estudio fue realizado con una combinación de datos de campo obtenidos entre 2002 y 2019, en las localidades de la Reserva Forestal de Colosó en el departamento de Sucre, a lo largo del arroyo Colosó, en la Serranía de la Coraza, desde el alto de Coraza hasta el nacimiento del arroyo grande de Coloso - Montes de María, ubicado a 9 930’ N y $75^{\circ} 21^{\prime}$ O (IGAC, 1969; Ministerio del Medio Ambiente, 2002) y fragmentos de Bosque seco Tropical (BsT) del departamento del Atlántico en los municipios de Piojó $10^{\circ} 75^{\prime} 69.53^{\prime \prime N}$ y $75^{\circ} 09 ' 68.56$ "O, Tubará a $10^{\circ} 47>37.68 \gg \mathrm{N}$ y $75^{\circ} 09>1,95 \gg \mathrm{O}$ y Usiacurí a $10^{\circ} 45^{\prime} 27.8^{\prime \prime}$ y $75^{\circ} 01^{\prime} 59,0$ "O. Aquí se ubican las áreas de bosques protegidos más grandes y en mejor estado de conservación con características de paisaje similares, ubicadas a lo largo de microcuencas o arroyos, con una altitud máxima entre los 200 y $330 \mathrm{msnm}$, temperatura media anual de $25^{\circ} \mathrm{C}$, con humedad relativa del $84 \%$ y precipitación media anual de 1.296,9 $\mathrm{mm}$, características que van de mayo a noviembre, alternadas con una estación seca entre diciembre y abril (IDEAM, 2010).

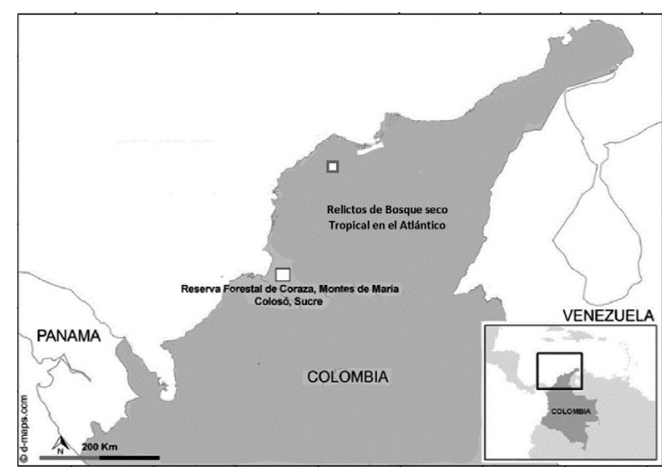

Figura 1. Ubicación de las localidades donde se describió la biología de polinización de Mucuna mutisiana y se registró la visita de sus polinizadores al norte de Colombia.

\section{Caracterización de la biología floral de M. mutisiana}

Los muestreos se realizaron en dos fragmentos del BsT en cada una de las localidades mencionadas, donde se registraron todas las plantas de $M$. mutisiana (Figura 1). Esta zona cuenta con paisajes en diferentes estados de conservación, en su mayoría constituidos por bosque higrotropofítico. 
Debido a que la planta habita principalmente en bosques de galería, se ubicaron transectos a lo largo de arroyos en los cuales se seleccionaron siete plantas o grupos de plantas que se definieron como estaciones de muestreo. En cada una de estas se colectaron veinticinco flores maduras que luego permitieron la descripción floral, el material fresco se preservó en solución FAA. El análisis morfológico de las flores se basó en los tipos florales propuestos por Nassar et al. (1997), las cuales corresponden a número de inflorescencias y número de flores por inflorescencia. A continuación, se realizó la caracterización morfológica en máximo cien flores en antesis de 25 individuos, se tomaron las medidas morfológicas de las flores como: longitud externa, longitud interna, largo de las anteras y largo del estilo, se determinó que se encuentran insertados en el receptáculo al extremo del pedicelo (Figura 2). La orientación de la flor se determinó definiendo dos criterios: (1) Vertical hacia arriba, cuando la flor se orienta en el sentido de la fuerza de la gravedad y la abertura de la corola se encuentra opuesta a la superficie del suelo. (2) Horizontal, cuando la flor se orienta de forma perpendicular en el sentido de la fuerza de gravedad. Tambien se registraron las siguientes características: número de estambres florales, número de óvulos por flor, y presencia o ausencia de olor floral.
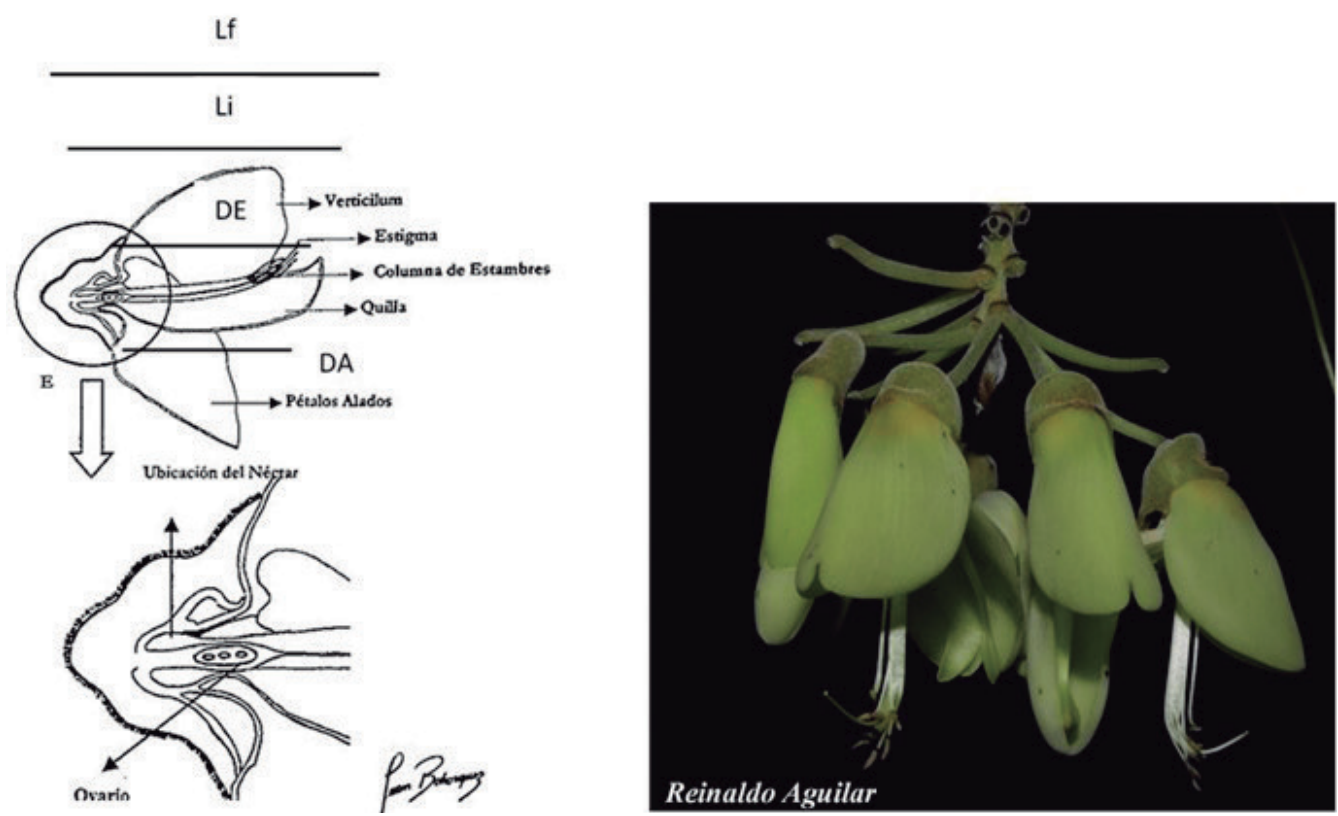

Figura 2. Corte transversal de una flor de Mucuna mutisiana. Medidas estimadas en las flores de Mucuna mutisiana; longitud de la flor (Lf), longitud interna (Li), longitud del pétalo de la quilla (Lpq), distancia de la antera a la base del tubo floral (DA), distancia del estigma a la base del tubo floral (DE). Ilustración elaborada por Bohórquez J. C. 
Caracterización de la producción de néctar y concentración de azúcar: los datos fueron recolectados durante la temporada de floración de la especie que se estimó entre 15 a 20 días. Se monitorearon un total de 112 flores que corresponden a 57 inflorescencia de siete individuos en las siete estaciones de muestreo. Las observaciones se iniciaron por la tarde, alrededor de las 18:00 h. cuando las flores maduras estuvieran listas para la visita de los murciélagos. El volumen de la secreción del néctar se midió con una micropipeta graduada con una escala de 1-100 $\mu \mathrm{l}$, en flores previamente aisladas con bolsas de tela de malla para evitar la atracción de los murciélagos. El volumen ( $\mu l)$ de néctar fue monitoreado cada dos horas, así como la concentración (\%) de azúcar con un refractómetro Brix Con (o32 ATC, Brix, Tokio, Japón).

\section{Observaciones de los visitantes florales} de $\boldsymbol{M}$. mutisiana: la interacción de la polinización entre los murciélagos con las flores de la especie Mucuna mutisiana fue monitoreada mediante el registro de visitas florales, los patrones de visita y el comportamiento de los visitantes diurnos y nocturnos. Para su identificación se realizaron capturas de los murciélagos en cada una de las siete estaciones, utilizando de tres a cuatro redes de niebla entre 12 , 6 y $4 \mathrm{~m}$ de ancho y $2,5 \mathrm{~m}$ de alto. Una vez capturados los murciélagos se registraron todas las mediciones morfológicas para su identificación taxonómica (Emmons, L. H. y Feer, F. 1999, Díaz, M.M., et al. 2016, Tirira, D. G. y S. F. Burneo 2012) y se procedió al marcaje de los especímenes capturados por medio del sistema de collar para su posterior recaptura. Con el fin de asegurar que los murciélagos sí habían visitado las flores, se verificó por observación directa la deposición del polen en el pelaje de los murciélagos, que posteriormente fue recolectado e identificado (Erdtman G. 1952). Se monitorearon entre 55 y 60 flores por noche, por observación directa apoyada por una cámara de video $(4 \mathrm{~K} \mathrm{AX} 43$, Handycam), a una distancia de 2 a $3 \mathrm{~m}$ de las inflorescencias por la dificultad para acceder a ellas, durante aproximadamente 20 días de floración. Se calculó la frecuencia de visitas de los potenciales polinizadores, principalmente los murciélagos y se describió el comportamiento de todos los animales, diurnos y nocturnos, que visitan las flores mediante la categorización según el nivel y tipo de interacción que se observó en las flores monitoreadas: (a) apertura explosiva sin daño a la flor (es decir, apertura exitosa); (b) néctar robado de una flor sin abrir; (c) destrucción de la flor (arrancada, mordida o caída) sin abrir (Kobayashi et al., 2015).

Análisis de datos: para evaluar la variación en la fenología reproductiva, tal como producción de flores (número de racimos y flores por planta) se implementó la estadística descriptiva. Se compararon las variables volumen del néctar, concentración de azúcar en el néctar y visitas florales; estas se trataron con el análisis ANOVA de una vía. La frecuencia de visitas de los murciélagos a las flores se calculó con el número total de visitas de murciélago por flor, para un número total de visita de flores por noche. Para 
comparar las diferencias en el volumen del néctar y la concentración de azúcar se realizó una prueba de Fisher en los diferentes muestreos por noche. Todos los análisis se realizaron utilizando el software Rstudio 2.15.

\section{Resultados y Discusión}

Biología floral: la época de floración de M. mutisiana ocurrió entre los meses de marzo y mayo y de octubre hasta noviembre, su duración aproximada fue de ocho a 14 semanas en cada época. Se registró un promedio de 57,3 a 108,8 racimos florales por planta $(\mathrm{SD}=51.5 ; \mathrm{n}=10)$. La mayor producción de flores fue entre la segunda y tercera semana de la floración con un total de cinco a seis flores maduras abiertas por noche por racimo y se obtuvo un promedio de 30,2 flores por individuo $(\mathrm{SD}=26,7 ; \mathrm{n}=10)$ con una producción total de 3420 flores $(\mathrm{SD}=3025.7 ; \mathrm{n}=10)$ cada noche para todas las estaciones.

La antesis de las flores ocurrió en el anochecer a partir de las 18:0o h, y estuvieron abiertas durante máximo dos noches; si no fueron polinizadas, posteriormente se caen, durante casi todo este tiempo se mantuvo la turgencia de las estructuras reproductivas. Las inflorescencias axilares y pseudoracemosas cuelgan desde el dosel en pedúnculos que van de 2,5 $\mathrm{m}$ a 3,5 $\mathrm{m}$ de longitud, formando racimos densos, los brotes florales más bajos o externos fueron los primeros en abrir y el ápice de la inflorescencia siguió creciendo, desarrollando brotes nuevos con 20 a 25 brotes florales $(n=10)$. Las flores son hermafroditas, presentando dirección hacia abajo, tipo papilionáceo, estructuralmente zigomórfico con simetría dorsoventral (Figura 2). Las dimensiones morfológicas estudiadas (Tabla 1) indican que las flores presentan una longitud de 6,3 $\mathrm{cm}(\mathrm{SD}=0.18, \mathrm{~N}=25)$, el diámetro promedio del pedicelo de $0,631 \mathrm{~cm}$, la corola es color crema y varía entre verde a blanca en flores maduras, además son pentafoliadas, con dos pétalos inferiores fusionados por sus márgenes adyacentes, constituidas por un par de pétalos conformados por la quilla, de $4,3 \mathrm{~cm}$ de longitud $(\mathrm{SD}=0.15, \mathrm{n}=25)$, en su interior se encuentran encerrados en promedio nueve estambres $(n=25)$, ocho fusionados y uno libre, de entre 3,4 y $4,3 \mathrm{~cm}$ de largo y el pistilo entre 4,3 y $5,5 \mathrm{~cm}(\mathrm{n}=25)$ de longitud. Además, la corola presentó dos pétalos alados laterales con longitud de $5,2 \mathrm{~cm}(\mathrm{SD}=0.2, \mathrm{n}=25)$ y un quinto pétalo superior llamado verticilum o estandarte, orientados hacia arriba con longitud de $4,2 \mathrm{~cm}(\mathrm{SD}=0.2, \mathrm{n}=25)$ (Figura 2). El olor del néctar en flores maduras fue fuerte, similar a un vegetal en descomposición (rancio o fermentado) $(n=10)$. Por su parte, los ovarios presentaron tres óvulos $(\mathrm{n}=25)$.

\section{Producción de néctar de las flores y} concentración de azúcares: la secreción del néctar comienza antes de la antesis, cuando las flores alanzan su madurez. Cuando las flores se abrieron, liberaron el máximo volumen del néctar registrado a las 18:0o h con 8,6 $\mu \mathrm{ll}(\mathrm{SD}=8,08 ; \mathrm{n}=112)$, luego decreció a las 19:00 h hasta 3,65 $\mu \mathrm{l}(\mathrm{SD}=4.0 ; \mathrm{n}=112)$, y el mínimo registro de producción de néctar se observó a 
Tabla 1. Descripción de las medias y la desviación estándar de las características morfológicas de 100 flores de 25 individuos de Mucuna mutisiana (Kunth) DC. Las flores presentaron una longitud máxima de 6,3 y una mínima de 4,2 cm.

\begin{tabular}{ccc}
\hline Característica morfológica de la flor & Promedio (cm) & Desviación estándar \\
\hline Longitud de la flor & 6.33 & 0.18 \\
\hline Longitud interna & 4.56 & 0.15 \\
\hline Longitud del pétalo de la quilla & 4.32 & 0.13 \\
\hline Distancia de la antera a la base del tubo floral & 3.4 & 0.22 \\
\hline Distancia del estigma a la base del tubo floral & 4.0 & 0.13 \\
\hline
\end{tabular}

las 22:00 h con 1,45 $\mu \mathrm{l}(\mathrm{SD}=1.6 ; \mathrm{n}=112)$, noche. La concentración de azúcar en el alcanzando valores cercanos a cero a la néctar mantuvo un promedio de $1,95^{\circ} \mathrm{Brix}$ 23:0o h. El total de producción de néctar $\quad(\mathrm{SD}=1.51 ; \mathrm{n}=112)$, el mayor porcentaje fue por flor obtenido cada noche fue de 18,58 de $4,68^{\circ} \mathrm{Brix}(\mathrm{SD}=5.03 ; \mathrm{n}=112)$ a las 18:00 $\mathrm{h}$, $\mu \mathrm{l}(\mathrm{SD}=18.74 ; \mathrm{n}=112)$ y el valor estimado posteriormente, disminuyó la producción de la producción de néctar por planta y se mantuvo fluctuando alrededor de por noche se estimó en $5,4 \mathrm{ml}$ para el $\operatorname{los} 2,29{ }^{\circ}$ Brix $(\mathrm{SD}=8.2 ; \mathrm{n}=112)$ (Figura número total de flores abiertas por 3). La prueba HSD de Tukey indicó que

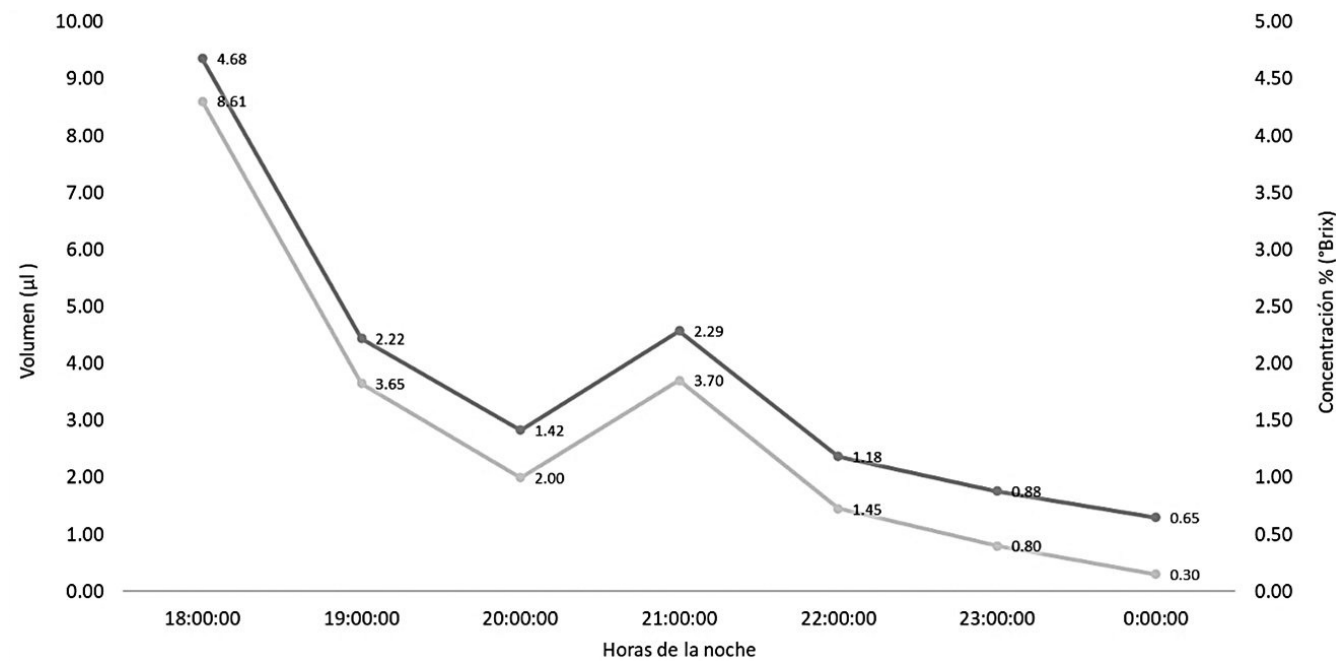

Figura 3. Promedio de la producción de néctar $(\mu \mathrm{L})$ y de la concentración de azúcares $\left({ }^{\circ}\right.$ Brix) en Mucuna mutisiana (Kunth) DC., $(\mathrm{n}=112)$ por cada hora durante la noche. 
existieron diferencias significativas entre los valores de disponibilidad media de néctar y la concentración de azúcares entre mediciones durante la noche. Se observó una variación significativa en la producción de néctar durante la noche $\left(\mathrm{F}_{6,777}=173,4 \mathrm{O}, \mathrm{P}=\right.$ 0.0001).

Visitantes florales de M. mutisiana: se identificaron tres clases de visitantes florales que corresponden a visitantes nocturnos, como algunos insectos de la familia Sphingidae que se posaron sobre los pétalos y la base de la corola sin tocar las anteras ni el estigma, también se observaron algunos mamíferos y otros vertebrados diurnos como aves.

Los mamíferos que visitaron las flores de M. mutisiana fueron los quirópteros Glossophaga longirostris (Miller, 1898) Glossophaga soricina (Pallas, 1766) (Figura 4); ambos de la familia Phillostomidae. La zarigüeya Caluromys lanatus (Olfers, 1818) (familia: Didelphidae). Las especies de murciélagos capturados con polen de $M$. mutisiana se separaron taxonómicamente según las medidas de los ejemplares, como fue el caso de la longitud del antebrazo que suele ser mayor en G. soricina, su hocico es más corto que la caja craneana, de cola corta que no supera la mitad de la longitud del uropatagio, contrario a $G$. longirostris que posee un hocico alargado y la longitud de su rostro suele ser mayor o igual a la caja craneana (Díaz, M.M., et al. 2016; Tirira, D. G. y S. F. Burneo, 2012). Las visitas de los quirópteros a las flores de $M$. mutisiana inician al atardecer y su mayor actividad se registró entre las 18:0o h y las 24:0o h. El mayor porcentaje de frecuencia de visita fue de $34,25 \%(\mathrm{SD}=39.8 ; \mathrm{n}=112)$ desde las 19:0o h hasta las 21:0o h (Figura 5). La prueba HSD de Tukey indicó que existieron diferencias significativas entre las medias de visitas a las flores por parte de los quirópteros.
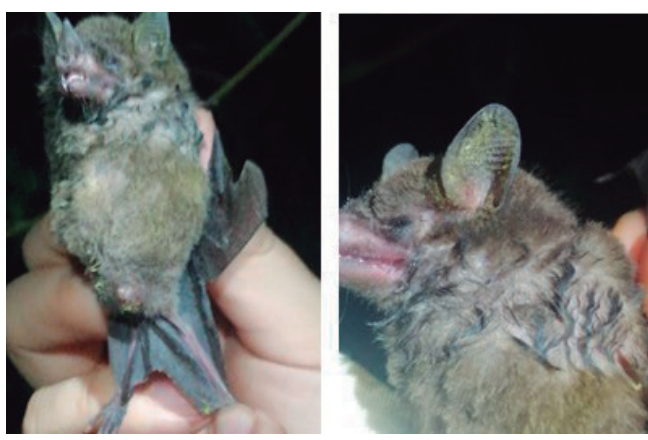

Figura 4. Visitantes florales de M. mutisiana a: Glossophaga soricina con polen de M. mutisiana. Elaboración propia.

Las visitas de los quirópteros a las flores de M. mutisiana son cortas $<2 \mathrm{~s}(\mathrm{n}=24)$, las dos especies registradas forrajean solitarias; cuando visitan las flores revolotean alrededor de ellas, luego los murciélagos introducen el rostro por la abertura de la corola a una alta velocidad, el movimiento brusco ocasiona que se active en la flor un mecanismo de elevación de la columna de estambres encerrados en los pétalos de la quilla inicialmente fusionados, lo que conlleva a que se presente un descenso de la quilla y el levantamiento permanente del verticilum, así el murciélago alcanza néctar en la base de la flor con su lengua y, posteriormente, se posa sobre las flores agarrándose con el pulgar del verticilum de la flor como soporte en la toma del néctar (Figura 6). 
Los quirópteros contactan su región ventral con el androceo y el gineceo y se impregnan de polen en el pelo del vientre, el uropatagio y las patas. Respecto a la actividad de las zarigüeyas, estas visitaron las flores alimentándose de ellas destruyéndolas. Durante la época de floración se capturaron 51 quirópteros correspondientes al género Glossophaga, de los cuales se marcaron 20 individuos de Glossophaga soricina y 11 de Glossophaga longirostris con collar plástico de un color para cada especie (Estrada-Villegas et al., 2010). En las muestras del pelaje y las heces de estos murciélagos se reconocieron tres tipos de dieta durante las épocas de floración, como frutos y semillas con 34\%, polen y néctar con $38,2 \%$ e insectos con $27,6 \%$.

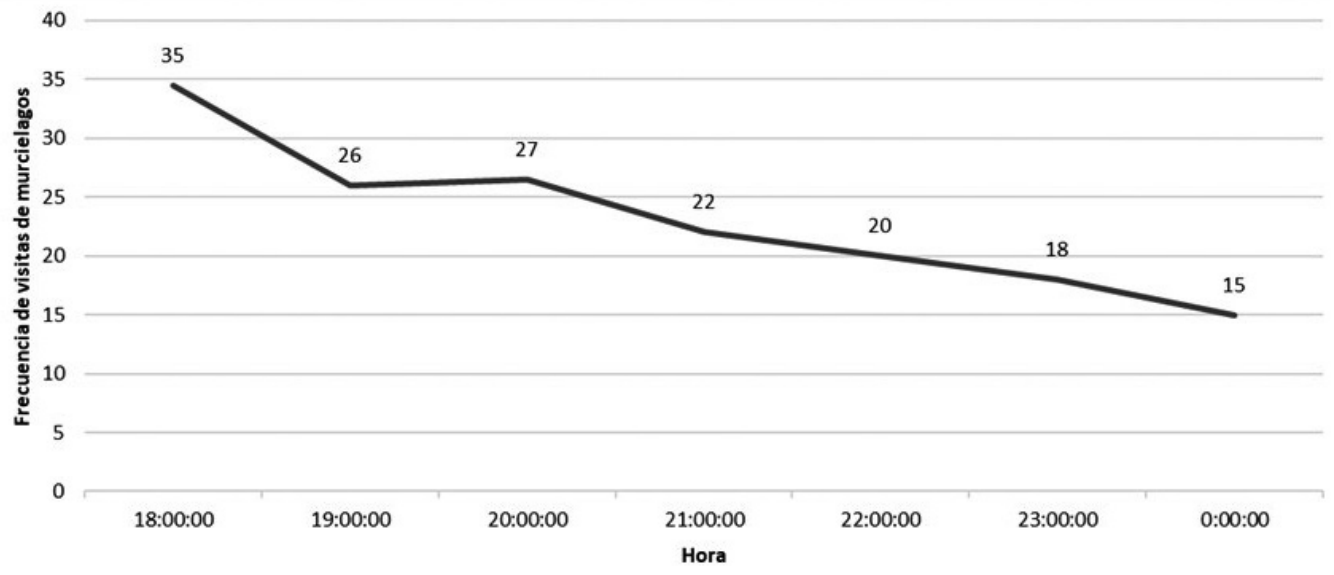

Figura 5. Frecuencias de visitas de murciélagos a las flores de M. mutisiana $(\mathrm{n}=10)$ en horas de la noche.

Las visitas exitosas durante la apertura de las flores y liberación del polen son realizadas principalmente por lo quirópteros; aquí también se reporta que otras especies animales interactuaron con las flores de M. mutisiana con un comportamiento de visita tipo robador de néctar de flores sin abrir y flores ya explotadas por los murciélagos. Como es el caso de las aves pertenecen a la familia Trochilidae, de las cuales se identificaron dos especies: Anthracothorax nigricollis (Vieillot, 1817) y Phaethornis anthophilus (Bourcier, 1843).
Los colibríes iniciaron la actividad como robadores de néctar de las flores de $M$. mutisiana a partir de las 7:0o h hasta la tarde, después de las 17:0o h. Su comportamiento de visita consistió en permanecer en vuelo suspendido frente a las flores por un promedio de 30 segundos a 1 minuto por flor $(\mathrm{SD}=0,56$ seg.; $n=10)$. Durante el forrajeo revolotearon alrededor de las flores cerradas, con el pétalo verticilum aun cerrado, robando flores maduras con el verticilum levantado dispuestas para su polinización, introduciendo su pico a 

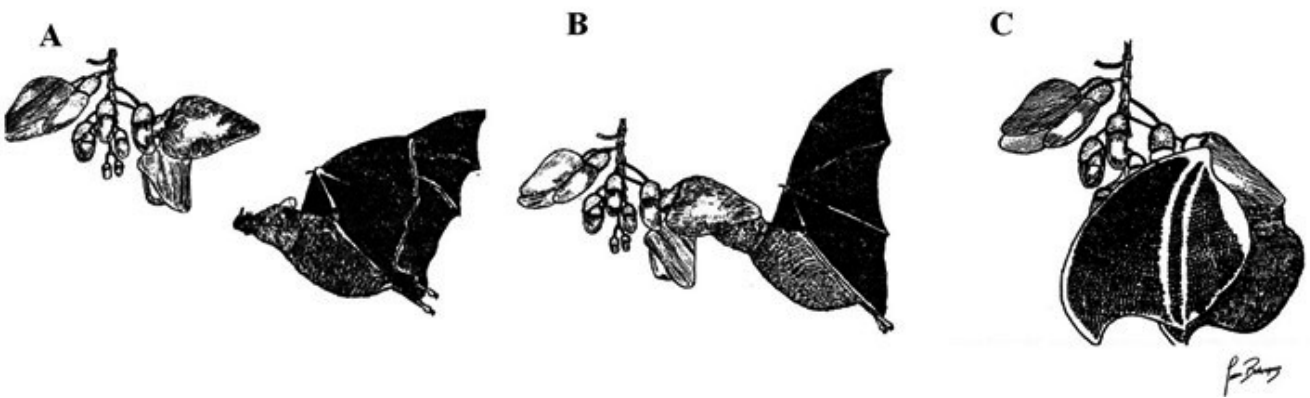

Figura 6. Visita de los quirópteros Glossophaga soricina y Glossophaga longirostris a las flores de M. mutisiana (A). Los quirópteros revolotean alrededor de ellas, (B) Introducen el rostro por la apertura de la corola a una alta velocidad, $(\mathrm{C})$ con su lengua alcanzan el néctar en la base de la flor posándose sobre ellas, empujando los pétalos de la quilla y separándolos, permitiendo que de manera explosiva se libere el polen. Ilustración elaborada por Bohórquez J. C.

través de este pétalo para tomar el néctar sin contacto con las partes reproductivas de las flores, actuando como polinizador no potencial de las flores de M. mutisiana.

\section{Discusión}

Los resultados confirman que las flores de M. mutisiana son abiertas y polinizadas con éxito por $G$. soricina y $G$. longirostris. Las observaciones revelaron que el pistilo y los estambres se ubican en contacto directo con el vientre de estos animales, que actúan como abridores explosivos, lo que sugiere que ambas especies son polinizadores eficaces. Esto lo respalda la observación hecha a las flores que fueron aisladas al inicio de la floración y que no realizaron autopolinización espontánea y, por tanto, no registraron formación de frutos. Por otro lado, los rasgos florales, las señales olfativas y visuales, así como la forma y dimensión de las partes florales, generan una recompensa química (composición de azúcar y concentración del néctar), lo cual encaja en los supuestos de la teoría del síndrome de polinización por quiropterofilia, permitiendo la atracción de sus visitantes potenciales.

La fenología de $M$. mutisiana es al final de la estación seca y principio de la estación lluviosa, desde marzo hasta junio y entre septiembre y noviembre, elemento característico en las plantas de este género (Moura et al. 2018; Sazima 1999). Los máximos picos de floración se presentan en el periodo intenso de lluvias, el cual sugiere que al parecer, está es estimulada por noches con temperaturas bajas de hasta $21^{\circ}$, lo cual coincide con las observaciones realizadas en localidades de tierras bajas de BsT y tierras altas, particularmente del bosque húmedo en Costa Rica (Heithaus, Fleming, y Opler 1975; T. M. Moura et al. 2013), donde se observó que los picos de floración de estas plantas polinizadas por murciélagos coinciden con las observaciones de este estudio. 
Indicios como la antesis crepuscular, que se da a partir de las 17:0o h; el volumen y la concentración de azúcares del néctar, que alcanza su máximo valor durante la noche después de las 18:00 h y disminuyen después de las 23:0o h, son características predecibles de las flores para atraer principalmente a polinizadores nocturnos (Muchhala 2003; Tschapka y von Helversen 2007; Willmer 2011).

El color verde-blanco o crema de la corola de M. mutisiana se relaciona con los síndromes florales de la quiropterofilia (rasgos florarles que permiten la atracción de murciélagos) y la falenofilia (conjunto de rasgos florales cuyo objeto es atraer a las palomillas o mariposas nocturnas), sin embargo, el olor a vegetal en descomposición, con pedúnculos colgantes de hasta $2 \mathrm{~m}$ en sus flores, está asociado al síndrome floral de la quiropterofília (Aguilar et al. 2015; Faegri y Pijl 1979; Fenster et al. 2004; Fleming, Geiselman, y Kress 2009; Tschapka y von Helversen 2007). Las plantas que presentan corolas anchas de aproximadamente $3 \mathrm{~cm}$ de diámetro y color externo poco atractivo, como en el caso de M. mutisiana, son mejor polinizadas por los murciélagos, al permitir que los hocicos alargados de estos animales penetren la corola de las flores. A diferencia de aquellas flores con corolas estrechas y colores vistosos, que son efectivamente polinizadas por los colibríes, pues permiten que su pico ingrese al interior de la flor. Esto evidencia un contraste entre las flores grandes bajas en pigmentos de antocianina y carotenoides, con respecto a las flores ricas en néctar con alto contenido de antocianinas, rasgos florales que confieren especificidad a los polinizadores, además de contribuir al aislamiento reproductivo prematuro de las plantas. Todo lo anterior sugiere que un mal ajuste en el ángulo de entrada a las flores o un cambio en la pigmentación de la corola podría derivar en una disminución de la efectividad en la transferencia de polen (Bradshaw y Schemske 2003; Schemske y Bradshaw 1999; Smith y Goldberg 2015). El pedicelo, de aproximadamente de $6,3 \mathrm{~mm}$ de diámetro en las flores de M. mutisiana puede soportar tanto el peso de la flor como el de su polinizador, esto evita que se rompa ante el fuerte movimiento brusco de los murciélagos nectarívoros hallados en los bosques secos del norte de Suramérica.

Se documentó una relación estrecha entre los murciélagos G. soricina, G. longirostris y L. curasoae con cactus columnares (Sánchez et al. 2007), según reportes hechos en Centro América, los dos primeros frecuentan las flores de M. andreana y posiblemente $M$. mutisiana (Baker 1970), los adultos de estos murciélagos pesan en promedio 12,4g a $14 \mathrm{~g}$ (Arias, Cadenillas, y Pacheco 2011), por lo tanto, una flor que presente un pedicelo con menor diámetro al registrado para las flores de M. mutisiana se podría romper al ser vistada por un polinizador con un peso de esta magnitud (Muchhala 2003). Además, los resultados de este estudio sugieren que estas plantas atraen a los visitantes nocturnos con guías de orientación, posiblemente de ecolocalización, como se reportó en $M$. holtoni (Heithaus 1982).

Algunas plantas adaptan sus rasgos florales a los polinizadores en cada región (Brian, James y Schwagerl, 2004; 
Duffy y Johnson, 2017; Westoby y Wright, 2006). Los resultados muestran que las estructuras de las flores de M. mutisiana varían ligeramente en su longitud con respecto a las flores de otras especies polinizadas por aves o por mamíferos no voladores que se distribuyen en otras regiones, como es el caso de M. sloanei y M. macrocarpa, respectivamente (Agostini et al., 2011; Kobayashi, S. et al., 2018). Se evidenció que $M$. mutisiana posee inflorescencias flageliformes que cuelgan hasta casi $2 \mathrm{~m}$ por debajo del follaje, con flores muy resistentes al contacto y su longitud de hasta de $6,3 \mathrm{~cm}(\mathrm{SD}=0.18$, $\mathrm{N}=25$ ). A diferencia de las observaciones realizadas en $M$. macrocarpa cuyas flores tienen mayor longitud (entre 65,0 \pm 2.7 hasta $76,7 \pm 2.3)$ y es polinizada por ardillas, zorros voladores y macacos en islas subtropicales y templadas en Asia. Además, se diferencian de Mucuna sloanei y Mucuna jaripa en Brasil (Agostini, 2004), que poseen flores en ambos casos de hasta $6,0 \mathrm{~cm} \pm 1,5 \mathrm{~cm}$ y sus polinizadores efectivos son tres especies de aves: Cacicus haemorrhous (Icteridae), Psarocolius decumanus (Icteridae) y Coereba flaveola (Thraupidae), las que, al parecer, varían en los rasgos de atractivo floral adaptados a la polinización ornitófila (Agostini et al., 2011). Aun así, las especies de este género que se conocen en el neotrópico son polinizadas por murciélagos, y que difieren ligeramente en el tamaño de la flor y los pétalos, por ejemplo: $M$. holtonii posee flores que varían entre 4,0 cm - 4,5 $\mathrm{cm}$ de longitud, $M$. urens presenta flores de entre $3,5 \mathrm{~cm}$ hasta $4,0 \mathrm{~cm}$ de longitud y M. mollis con flores entre $4,0 \mathrm{~cm}$ a 5,0 cm de longitud. Estos datos sugieren que para que un visitante floral pueda realizar la apertura explosiva de una flor, este debe ajustarse al tamaño de la misma para que la polinización tenga éxito.

Los visitantes florales que con mayor frecuencia contactan el androceo y el gineceo de las flores de $M$. mutisiana son los murciélagos G. soricina y G. longirostris, por lo que se podrían considerar como posibles polinizadores de esta liana que se encuentra en los BsT del norte de Suramérica y en parte de Centro América, alrededor de los bosques de galería, desde el nivel del mar hasta 2500 m (T. M. D. Moura et al., 2018). Durante los meses de floración, $G$. soricina y $G$. longirostris visitan de con alta frecuencia las flores de M. mutisiana, por lo que en la época de floración de esta planta son visitantes florales que comparten su recurso. Durante los meses que no florece M. mutisiana, las especies $G$. soricina y G. longirostris explotan otros recursos alimenticios como frutas e insectos. Algunos estudios sustentan que estas especies poseen la capacidad de utilizar los recursos ofrecidos por el entorno, como por ejemplo, flores de cactus columnares que suele influir sobre estructura del ensamblaje de los murciélagos en el BsT del neotrópico (Sánchez et al. 2007).

Otros autores reportan que los murciélagos representan ventajas como polinizadores, dada sus dimensiones corporales, la presencia de pelo y que cuentan con una excelente memoria espacial (Tschapka y von Helversen, 2007). Durante todo su 
período de floración se evidencia una alta frecuencia de visitas a las plantas para la explotación del néctar, lo cual genera un impacto positivo sobre la reproducción de las plantas; además, son capaces de transportar grandes cantidades de polen a largas distancias (Fleming, et. al 2009; Heithaus et. al 1975). Se ha reportado que los murciélagos Glossophaginos presentan un alto valor en el índice de relación de aspecto con alas grandes y delgadas que les permite volar más rápido y, tal vez, recorrer mayores distancias (Tschapka 2004). Estas características aumentan la posibilidad de trasladar el polen a largas distancias, de esta forma, cuando el murciélago toma originalmente el polen podría trasladarlo a otro individuo de M. mutisiana, reduciendo la posibilidad de encontrar una planta emparentada, lo que podría favorecer la xenogamia. Los troquílidos que visitan las lianas M. mutisiana tienen un pico recto que penetran por la parte superior del verticilum llegando al nectario en flores que permanecen cerradas y es posible que actúen como robadores de néctar, como fue observado en este estudio.

Las características del néctar producido por M. mutisiana, que es polinizada por murciélagos glossophaginos en el neotrópico, es menor que entre las especies asiáticas, como es el caso de $M$. macrocarpa, principalmente polinizadas por macacos (Muscata Macaca) en Kyushu, Japón (Kobayashi et al., 2015), por zorros voladores (Pteropus dasymallus) en Okinawa, Japón (Kobayashi, Denda, et al., 2018), y por Ardillas Callosciurus en Taiwán y Tailandia (Kobayashi et al., 2017;
Kobayashi, Denda, et al., 2019); asimismo, M. championi principalmente polinizada por un roedor (Niviventer fulvescens) y el murciélago de la fruta de nariz corta, un megaquiróptero (Cynopterus sphinx) en Hong Kong (Kobayashi et al., 2021).

Poco se conoce de las características específicas del néctar de las especies de mucuna polinizadas por mamíferos y distribuidas en el neotrópico, no obstante, M. mutisiana posee una relativa variabilidad en el volumen del néctar con $8,6 \pm 0,7(\mu \mathrm{l})$ y concentración de azúcar en $4,68 \% \pm 1,3 \%$, a diferencia de las especies que se distribuyen en otras regiones, como es el caso de M. championi con un mayor volumen del néctar con 256,2 $\pm 65,0(\mu \mathrm{l})$ y concentración de azúcar en 24,68\% $\pm 1,7 \%$ (Kobayashi et al., 2021). M. sempervirens, cuya concentración de azúcar en el néctar es 293,8 \pm 15,0 ( $\mu$ l) (Liu et al., 2013) y finalmente M. birdwoodiana con el mayor volumen del néctar, de 882.9.2 $\pm 75, \mathrm{O}(\mu \mathrm{l})$ y concentración de azúcar en $24,3 \% \pm 0,6$ \% (Kobayashi et al., 2021). Estos datos sugieren que, para que un visitante floral pueda explorar las flores, se requiere un nivel de ajuste en la recompensa a los polinizadores entre las diversas especies de Mucuna. Al documentar la biología de polinización de $M$. mutisiana, se halló un alto valor puesto que permite documentar la interacción entre las especies de este género con sus potenciales polinizadores, que actúan como abridores de apertura explosiva. Cabe señalar que se conoce poco sobre las características específicas de recompensa de las especies de este 
género polinizadas por mamíferos y que se encuentran distribuidas en el neotrópico.

\section{Conclusiones}

M. mutisiana presenta adaptaciones del síndrome floral de quiropterofilia en el BsT al norte de Colombia. Las flores de esta enredadera requieren una fuerza mecánica para su apertura, lo cual es una adaptación orientada a mamíferos voladores de exploración explosiva, evitando ladrones potenciales de néctar con masa corporal más pequeña que la de sus potenciales polinizadores. Las flores de mucuna presentan adaptaciones con estrategias para atraer polinizadores nocturnos a través de guía acústica para murciélagos polinizadores (von Helversen \& von Helversen, 1999), además, presentan patrones de secreción de néctar con concentraciones energéticas para polinizadores de murciélagos y aves, como sucede en otras especies del género (Agostini et al. 2011). Los resultados de esta investigación concuerdan con los obtenidos en estudios de plantas de este mismo género polinizadas por murciélagos en bosques tropicales de Brasil, con una producción energética que varió entre $28,7 \%$ hasta $39,8 \%$ ${ }^{\circ}$ Brix, con 5,41 ml de néctar producido por planta por noche (Agostini, Sazima, y Galetto 2011), al parecer, como atractivo adaptado para promover la visita de polinizadores nocturnos, lo cual es ventajoso, tanto para las plantas como para los murciélagos nectarívoros ya que permite a las plantas les reducir el riesgo de geitonogamia (Agostini, Sazima, y Galetto, 2011).
El tamaño del cuerpo y la tasa metabólica de estos murciélagos polinizadores está relacionada con la producción energética en el néctar de las flores, lo que implica una ganancia energética, ya que estos gastan cerca de $114 \mathrm{cal}$ en lugares que sobrepasan más de $10^{\circ} \mathrm{C}$, que corresponde aproximadamente al $1,2 \%$ del total de energía presupuestada para $24 \mathrm{~h}$ (Willmer 2011). En el caso de G. soricina y G. longirostris, registrados en este estudio, presentaron un peso de entre $8 \mathrm{~g}$ a $16 \mathrm{~g}$; su flexibilidad para alimentarse de manera oportunista durante el periodo de muestreo incluyó frutas e insectos durante las épocas del año con poca disponibilidad del néctar. Además, estudios han encontrado que $G$. soricina es capturada en mayor número en fragmentos y en islas dentro del bosque, por lo que es frecuente encontrarlo, tanto en paisajes fragmentados como en matriz de bosque conservado que ofrecen recursos alimenticio para estos murciélagos (Stefan et al. 2020)such as bats, the matrix is not necessarily an entirely in surmountable obstacle. We studied the effects of fragmentation on New-World leaf-nosed bats (Phyllostomidae. Según estos resultados, las características en la biología de polinización y de atractivo floral M. mutisiana en interacción con sus polinizadores puede relacionarse con las características de la fauna neotropical.

En general, las flores polinizadas por mamíferos son más grandes y robustas que las de plantas polinizadas por insectos, y estas flores a menudo tienen largos estambres y pistilos que se extienden 
más allá de la corola (Willmer, 2011). Los resultados de esta investigación muestran que las estructuras de las flores de $M$. mutisiana varían ligeramente en su longitud con las de flores de otras especies polinizadas por aves o por mamíferos no voladores, como es el caso de M. sloanei y M. macrocarpa respectivamente (Agostini et al., 2011; Kobayashi, S., et al., 2018), con flores muy resistentes al contacto y con longitud de hasta de $6,3 \mathrm{~cm}(\mathrm{SD}=$ $0.18, \mathrm{~N}=25$ ), a diferencia de las observaciones realizadas en $M$. macrocarpa cuyas flores tienen mayor longitud entre 65,0 \pm 2,7 hasta $76,7 \pm 2,3$, polinizada por ardillas, zorros voladores y macacos en islas subtropicales y templadas de Asia.

Además, se diferencian con Mucuna sloanei y Mucuna jaripa en Brasil (Agostini, 2004) en donde tres especies de aves actúan como polinizadoras efectivas y que, al parecer, varían en los rasgos de atractivo floral adaptados a la polinización ornitófila. Por lo tanto, se sugiere a futuro realizar estudios sobre los rasgos atractivos de las especies de este género, donde se incluyan los rasgos florales y quizás las adaptaciones que presentan sus flores al polinizador principal en cada región.

\section{Agradecimientos}

Agradezco a Idea Wild y Alaska Birds, por la financiación parcial y donación de equipos para el trabajo de campo, a la Corporación Regional de Sucre (Carsucre), a la Universidad del Atlántico, Facultad de Ciencias Básicas, Departamento de Biología y también a la Universidad del Norte, Departamento de Química y Biología.

\section{Referencias}

Agostini, K., Sazima, M. y Galetto, L. 2011. Nectar production dynamics and sugar composition in two Мucuna species (Leguminosae, Faboideae) with different specialized pollinators. Naturwissenschaften 98, 933. Disponible en: https:// doi.org/10.1007/soo114-011-0844-6 
Aguilar, R., Martén-Rodriguez, S., Avila-Sakar, G., Ashworth, L., Lopeazaraiza-Mikel, M., Lobo J.A., Marten-Rodrígez S., Fuchs E.J., Sánchez-Montoya G., Bernardello G. y Quesada, M. 2015. A global review of pollination syndromes: A response to Ollerton et al. 2015. Fournal of Pollination Ecology, 17, 126128. Disponible en: https://doi.org/10.26786/1920-7603(2015)21

Aguilar, R., Cristóbal-Pérez, E. J., Balvino-Olvera, F. J., de Jesús Aguilar-Aguilar, M., Aguirre-Acosta, N., Ashworth, L. LoboJ.A., Marten-Rodrígez S., Fuchs E.J., Sánchez-Montoya G., Bernardello G. y Quesada, M. 2019. Habitat fragmentation reduces plant progeny quality: a global synthesis. Ecology letters, 22(7), 1163-1173.Disponible en: https://doi.org/10.1111/ele.13272

Aguilar, Ramiro, Lorena Ashworth, Leonardo Galetto, y Marcelo Adrian Aizen. 2006. Plant Reproductive Susceptibility to Habitat Fragmentation: Review and Synthesis through a Meta-Analysis. Ecology Letters 9(8): 968-80.

Aldana-Domínguez, J., Montes, C., Martínez, M., Medina, N., Hahn, J., y Duque, M. 2017. Biodiversity and Ecosystem Services Knowledge in the Colombian Caribbean: Progress and Challenges. Tropical Conservation Science. Disponible en: https://doi.org/10.1177/1940082917714229

Arias, E., Cadenillas, R., \& Pacheco, V. 2009. Dieta de murciélagos nectarívoros del Parque Nacional Cerros de Amotape, Tumbes. Revista Peruana De Biología, 16(2), 187-190. Disponible en: https://doi.org/10.15381/rpb.v16i2.204

Ashman, T., Knight, T.M., Steets, J.A., Amarasekare, P., Burd, M., Campbell, D.R., Dudash, M.R.,Johnston, M.O., Mazer, S.J., Mitchell, R.J., Morgan, M.T. and Wilson, W.G. 2004. POLLEN LIMITATION OF PLANT REPRODUCTION: ECOLOGICAL AND EVOLUTIONARY CAUSES AND CONSEQUENCES. Ecology, 85: 2408-2421. Disponible en: https://doi.org/10.1890/03-8024

Baker, Herbert G. 1970. Two Cases o1 Bat Pollination in Central America. REVISTA DE BIOLOGÍA TROPICAL: 17: 187-197. 
Bradshaw, H. D., y Schemske, D. W. 2003. Allele substitution at a flower colour locus produces a pollinator shift in monkeyflowers. Nature, 426(6963), 176-178. Disponible en: https://doi.org/10.1038/natureo2106

Dunphy B.K., Hamrick J.L. y Schwagerl J. 2004. A comparison of direct and indirect measures of gene flow in the bat-pollinated tree Hymenaea courbaril in the dry forest life zone of southwestern Puerto Rico. International fournal of Plant Sciences, $165(3), 427-436$.

Carbone, L.M., Tavella, J., Pausas, J.G. y Aguilar, R. A. 2019. A global synthesis of fire effects on pollinators. Global Ecol Biogeogr. 2019; 28: 1487- 1498. Disponible en: https://doi. org/10.1111/geb.12939

Chacoff, N. P., Aizen, M. A. y Aschero, V. 2008. Proximity to forest edge does not affect crop production despite pollen limitationProc. R. Soc. B.275907-913. Disponible en: http://doi. org/10.1098/rspb.2007.1547

Cusser, S., Neff, J. L., y Jha, S. (2016). Natural land cover drives pollinator abundance and richness, leading to reductions in pollen limitation in cotton agroecosystems. Agriculture, Ecosystems \& Environment, 226, 33-42. Disponible en: https:// doi.org/10.1016/j.agee.2016.04.020

Díaz, M. M., Aguirre, L. F., y Barquez, R. M. 2011. Clave de identificación de los murciélagos del cono sur de Sudamérica. Centro de Estudios en Biología Teórica y Aplicada; 94 pp. Disponible en: http:/hdl.handle.net/11336/115357

Duffy, K. J. y Steven D. J. 2017. Specialized Mutualisms May Constrain the Geographical Distribution of Flowering Plants. Proceedings of the Royal Society B: Biological Sciences 284(1866): 20171841. Disponible en: https://doi.org/10.1098/rspb.2017.1841.

Erdtman G. 1952. Pollen Morphology and Plant Taxonomy: Angiosperms. Stockholm, Sweden. Almqvist and Wiksell. 553 p. 
Emmons, L. H. y Feer, F. 1999. Mamíferos de los bosques húmedos de América Tropical, una guía de campo. ıera edición en español. 1era edición en español. Editorial FAN. Santa Cruz de la Sierra, Bolivia.

Faegri, Knut, y L. van der Pijl. 1979. The Principles of Pollination Ecology. 3d rev. ed. Oxford ; New York: Pergamon Press.

Fenster, C. B., Scott, W., Wilson, P., Dudash, M. R. y Thomson, J. D. 2004. Pollination Syndromes and Floral Specialization. Annual Review of Ecology, Evolution, and Systematics 35(1): 375-403. Disponible en: https://doi.org/10.1146/annurev. ecolsys.34.011802.132347

Fleming, T. H., Geiselman, C., y Kress, W. J. 2009. «The Evolution of Bat Pollination: A Phylogenetic Perspective». Annals of Botany 104(6): 1017-1043. Disponible en: https://doi. org/10.1093/aob/mcp197

Heithaus, E. R. 1982. Coevolution between Bats and Plants. En Ecology of Bats, ed. Thomas H. Kunz. Boston, MA: Springer US, 327-367. Disponible en: https://doi.org/10.1007/978-14613-3421-7_9

Heithaus, E. R., Fleming, T. H. y Opler, P. A. 1975. Foraging Patterns and Resource Utilization in Seven Species of Bats in a Seasonal Tropical Forest. Ecology 56(4): 841-54.

Horner, M. A., Fleming, T. H. y Sahey, C. T. 1998. Foraging Behaviour and Energetics of a Nectar-Feeding Bat, Leptonycteris Curasoae (Chiroptera: Phyllostomidae). fournal of Zoology 244(4): 575-86.

Kobayashi, S., Denda, T., Mashiba, S., Iwamoto, T., Doi, T., y Izawa, M. 2015. Pollination Partners of Mucuna Macrocarpa (Fabaceae) at the Northern Limit of Its Range: Novel Explosive Openers of M. Macrocarpa. Plant Species Biology 30(4): 272-278. Disponible en: https://doi.org/10.1111/1442-1984.12065 
Kobayashi, S., Hirose, E., Denda, T. y Izawa M. 2018. Who Can Open the Flower? Assessment of the Flower Opening Force of Mammal-Pollinated Mucuna Macrocarpa: Operative Force of M. Macrocarpa. Plant Species Biology 33(4): 312-16. Diponible en: https://doi.org/10.1111/1442-1984.12221

Kobayashi, S., Gale, S.W., Denda, T. y Izawa, M. 2019. Civet pollination in Mucuna birdæoodiana (Fabaceae: Papilionoideae). Plant Ecol 220, 457-466. Disponible en: https://doi.org/10.1007/ S11258-019-00927-y

Kobayashi, S., Gale, S.W., Denda, T. y Izawa, M. 2021. Ratand bat-pollination of Mucuna championii (Fabaceae) in Hong Kong. Plant Species Biology, 36: 84- 93. https://doi. org/10.1111/1442-1984.12298

Liu, T., Shah, A., Zha, H. G., Mohsin, M. y Ishtiaq, M. 2013. Floral nectar composition of an outcrossing bean species mucuna sempervirens hemsl (fabaceae). Pakistan Fournal of Botany, $45(6), 2079-2084$.

Moura, T. M., Lewis, G. P., Mansano, V. F., y Tozzi, A. M. G. A. 2013. Three New Species of Mucuna (Leguminosae: Papilionoideae: Phaseoleae) from South America. Kew Bulletin 68(1): 143-50.

Moura, T. M., Lewis, G. P., Mansano, V. F. y Tozzi, A. M. 2018. A Revision of the Neotropical Mucuna Species (LeguminosaePapilionoideae). Phytotaxa 337(1): 1. Disponible en: https://doi. org/10.11646/phytotaxa.337.1.1

Muchhala, N. 2003. Exploring the Boundary between Pollination Syndromes: Bats and Hummingbirds as Pollinators of Burmeistera Cyclostigmata and B. Tenuiflora (Campanulaceae). Oecologia 134(3): 373-80. Disponible en: https://doi.org/10.1007/s00442-002-1132-o

Nassar J.M., Ramírez N. y Linares O. 1997. Comparative pollination biology of Venezuelan columnar cacti and the role of nectar-feeding bats in their sexual reproduction. American Journal of Botany 84(7):918-927. 
Pallas, P. S. 1766. Miscellanea zoologica quibus novae imprimis atque obscurae animalium species describuntur et observationibus iconibusque illustrantur. Hague Comitum: P. van Cleef. Disponible en:https://doi.org/10.5962/bhl.title.69851

Pizano, G. y García, H. 2014. El bosque seco tropical en Colombia. Instituto de Investigación de Recursos Biológicos Alexander Von Humboldt, Bogotá (Colombia) Ministerio de Ambiente y Desarrollo Sostenible, Bogotá (Colombia). 350 pp.

Sánchez, F., Alvarez, J., Ariza, C. y Cadena, A. 2007. Bat assemblage structure in two dry forests of Colombia: Composition, species richness, and relative abundance. Mammalian Biology, 72(2): 82-92. Disponible en: https://doi. org/10.1016/j.mambio.2006.08.003

Sazima, M., Buzato, S. y Sazima, I. 1999. Bat-pollinated flower assemblages and bat visitors at two Atlantic forest sites in Brazil. Annals of Botany, 83(6), 705-712.

Schemske, D. W. y Bradshaw, H. D., Jr. 1999. Pollinator preference and the evolution of floral traits in monkeyflowers (Mimulus). Proceedings of the National Academy of Sciences of the United States of America, 96(21), 11910-11915. Disponible en: https://doi.org/10.1073/pnas.96.21.11910.

Smith, S. D. y Goldberg, E. E. 2015. Tempo and mode of flower color evolution. American journal of botany, 102(7), 1014-1025. Disponible en: https://doi.org/10.3732/ajb.1500163

Stefan D. Brändel, S. D. Brändel, Thomas Hiller, T. Hiller, Tanja K. Halczok, T. K. Halczok, Gerald Kerth, G. Kerth, Rachel A. Page, R. A. Page y Marco Tschapka, M. Tschapka. 2020. Consequences of fragmentation for Neotropical bats: The importance of the matrix. Biological conservation, 252, 108792. Disponible en: 10.1016/j.biocon.2020.108792 
Tirira, D. G. y Burneo, S. F. (eds.). 2012. Investigación y conservación sobre murciélagos en el Ecuador. Pontificia Universidad Católica del Ecuador, Fundación Mamíferos y Conservación y Asociación Ecuatoriana de Mastozoología. Publicación especial sobre los mamíferos del Ecuador 9. Quito.

Tschapka, M., y Von Helversen, O. 2007. Phenology, nectar production and visitation behaviour of bats on the flowers of the bromeliad Werauhia gladioliflora in a Costa Rican lowland rain forest. Fournal of Tropical Ecology, 23(4), 385-395. Disponible en: https://doi.org/10.1017/So266467407004129

Voigt, C. C. 2004. The Power Requirements (Glossophaginae: Phyllostomidae) in Nectar-Feeding Bats for Clinging to Flowers. fournal of Comparative Physiology B. 174, 541-548 (2004). Disponible en: https://doi.org/10.1007/so0360-004-0442-4

Westoby, M., y Wright, I.J. 2006. Land-plant ecology on the basis of functional traits. Trends in ecology \& evolution, 21(5), 261-268.

Willmer, Pat. 2011. Pollination and Floral Ecology. Princeton, N.J: Princeton University Press. 